\title{
Real-time and non-invasive measurements of cell mechanical behaviour with optical coherence phase microscopy
}

\author{
D Gillies ${ }^{1}$, W Gamal ${ }^{2}$, A Downes ${ }^{1}$, Y Reinwald ${ }^{3}$, Y Yang ${ }^{3}$, A El Haj ${ }^{3}$, P O Bagnaninchi ${ }^{2}$
}

${ }^{1}$ Institute for Bioengineering, University of Edinburgh, Edinburgh, UK

${ }^{2}$ MRC Scottish Centre for Regenerative Medicine, University of Edinburgh, Edinburgh, UK

${ }^{3}$ Institute for Science and Technology in Medicine, Keele University, UK

\begin{abstract}
There is an unmet need in tissue engineering for non-invasive, label-free monitoring of cell mechanical behaviour in their physiological environment. Here, we describe a novel optical coherence phase microscopy (OCPM) set-up which can map relative cell mechanical behaviour in monolayers and 3D systems non-invasively, and in real-time. 3T3 and $\mathrm{MCF}-7$ cells were investigated, with $\mathrm{MCF}-7$ demonstrating an increased response to hydrostatic stimulus indicating

MCF-7 being softer than 3T3, demonstrating the ability to provide qualitative data on cell mechanical behaviour. Quantitative measurements of 6\% agarose beads have been taken with commercial Cell Scale Microsquisher ${ }^{\circledR}$ system demonstrating that their mechanical properties are in the same order of magnitude of cells, indicating that this is an appropriate test sample for the novel method desctibed.
\end{abstract}

\section{Keywords: $\mathbf{x x x x}$}

\section{INTRODUCTION}

The self-renewal and differentiation abilities of stem cells mean that they are vitally important in regenerative and therapeutic medicine. Mechanical stimuli have been shown to have a major role in regulating stem cell behaviour, with differentiation defined as a mechanosensitive process controlled by the stiffness of the stem cell substrate (Engler et al., 2006). The mechanical behaviour of cells from the nanoscopic to macroscopic scales is known to alter (Kennedy et al., 2014a), and nanoscale features have been shown to influence cell behaviour on synthetic surfaces (E. Kim et al., 2009). There is therefore a need to investigate the mechanical behaviour of the stem cell niche as well as its response to various mechanical stimuli. To address this need we have developed a novel non-invasive method to perform elastography on live cells at cellular resolution with an optical coherence phase microscopy (OCPM).

On the macroscopic scale, clinicians have used manual palpation of suspect tissues as a qualitative diagnostic tool for centuries. Imaging techniques, such as ultrasound and Magnetic Resonance Imaging (MRI) elastography have been introduced, however both lack the spatial resolution to be used on the cellular scale. The measurement of mechanical behaviour on the nano- and microscopic scale has used techniques such as atomic force microscopy (AFM), optical tweezers, and optical coherence elastography (OCE) (Kennedy et al., 2014b). AFM uses a cantilever and tip to determine quantitative cell mechanical properties, achieving high resolution and mechanical sensitivity. It does, however, suffer from the drawback of its inherent invasiveness. Compression OCE is comparable to palpation in that a force is applied to the sample under investigation and the resulting displacement is tracked. 
Optical coherence microscopy (OCM) can achieve sub-micron resolution with high dynamic range and sensitivity by combining OCT with high transverse resolution confocal microscopy, allowing for 3D cellular imaging. OCPM further extends OCM to measure the phase changes in a sample. OCPM can distinguish phase and cross sectional depth information from a sample. It is sensitive to sub-micrometer changes in optical path length (OPL), and achieves high spatial resolution. It is therefore an ideal candidate for monitoring motion. OCPM has been used to characterise nanoscale cellular dynamics events in live cells (Ellerbee et al., 2007), and has been shown to measure cell viability based on intracellular optical fluctuations (Holmes 2015, Bagnaninchi 2011)

\section{MATERIALS AND METHODS}

\subsection{Optical Coherence Phase Microscopy}

A commercial optical coherence tomography system (Callisto, Thorlabs) was coupled to the side port of an inverted fluorescent Leica DMIRE2 microscope. The superluminescent light source, centred at $930 \mathrm{~nm}$ with a FWHM of $90 \mathrm{~nm}$ provides an axial resolution of $5 \mu \mathrm{m}$ in water, while the lateral resolution is determined by the objective. The custom scanning head was able to operate in an ad-hoc scanning mode allowing the collection of the varying phase over time, and in depth into the sample, with increased phase stability due to the common path reference (Holmes et al., 2015).

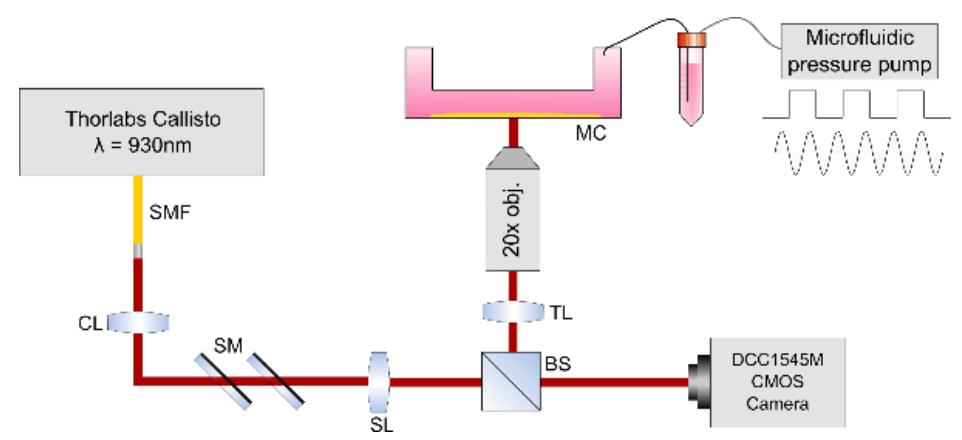

Figure 1: OCPM set-up for quantitative measurement of cell mechanical properties: SMF, single mode fibre; CL, collimating lens; SM, scanning mirrors; SL, scanning lens; BS, beam splitter; TL, tube lens; MC, microchannel.

Cells were exposed to cyclic mechanical stimuli inside the microchip ranging from $1,000 \mathrm{~N} \cdot \mathrm{m}^{-2}$ to $20,000 \mathrm{~N} \cdot \mathrm{m}^{-2}$ with a typical frequency of $300 \mathrm{mHz}$. A 4D data cube $(256 \mathrm{X} 256 \times 512 \times 36$ in $(\mathrm{X}, \mathrm{Y}, \mathrm{Z}, \mathrm{T})$ pixels) was then captured with an acquisition frequency of 1,200 A scans per second to sample the varying phase over time which was converted to displacement through the equation:

$$
d=\frac{\Delta \Phi \lambda_{0}}{4 \pi n}
$$

Where; $n$ is the refractive index, $\lambda_{0}$ is the central wavelength and $\Delta \Phi$ is the phase difference between adjacent B scans.

For a sinusoidal input pressure, the relative displacement of each pixel was then determined through the equation:

$$
\Delta d=2 . * \sqrt{2} . * \Delta \Phi R M S(x, y) \frac{\lambda_{0}}{4 \pi n}
$$

Where $\Delta \Phi R M S(x, y)$ is the phase root mean squared, $\lambda_{0}$ is the central wavelength, $n$ is the refractive index. 
And similarly for the case of a rectangular input pressure we have:

$\Delta d=2 * \Delta \Phi R M S(x, y) \frac{\lambda_{0}}{4 \pi n}$

2)

$\Delta \mathrm{P}$

Cyclic hydrostatic pressure

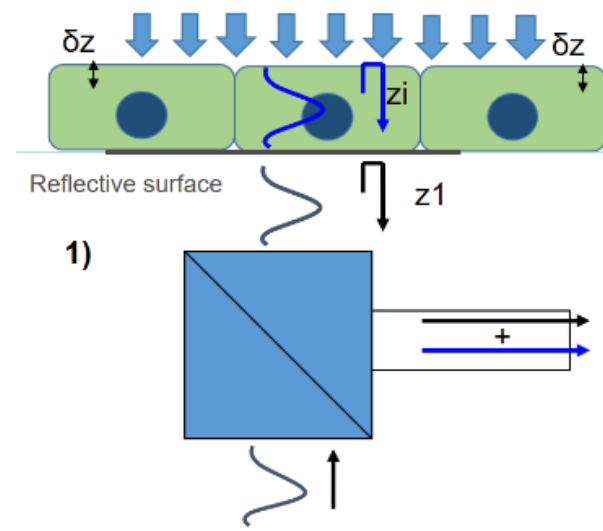

3)

Phase shift at zi

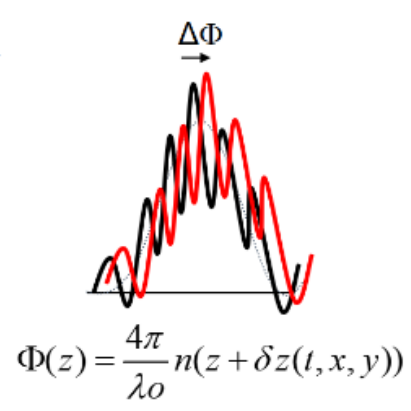

4)

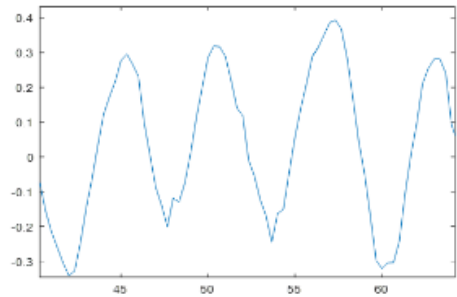

5)

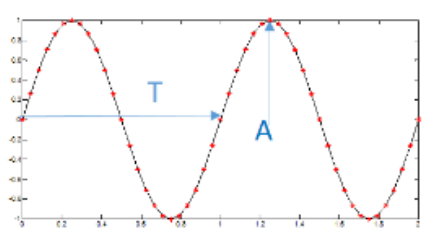

$\Delta \mathrm{d}=2 .{ }^{*} \sqrt{ } 2 .{ }^{*} \Delta \phi \mathrm{RMS}(\mathrm{x}, \mathrm{y}) . \lambda_{0} / 4 \pi \mathrm{n}$

Figure 2: Conversion of phase change into relative displacement. 1) Imaging system 2) Cyclic hydrostatic pressure is applied to cells which are cultured on a clear, reflective surface 3) The phase change is measured at each individual pixel 4) Displacement is measured 5) The relative change in displacement is calculated using the RMS.

\subsection{Cell Scale Microsquisher ${ }^{\circledR}$}

Agarose beads of size $150 \mu \mathrm{m}$ to $350 \mu \mathrm{m}$ were subjected to parallel plate compression in a water bath at a strain rate of $2.5 \mu \mathrm{m} / \mathrm{s}$ using the Cell Scale Microsquisher ${ }^{\circledR}$ and Squisherjoy software. Plate and cantilever dimensions were optimised for bead size. The force-displacement data was then converted into stress-strain, with the associated curve used to obtain a linear regression line from which the elasticity was calculated at $10 \%$ nominal compression of the sphere.

\subsection{Cell Culture}

Breast cancer cells (MCF-7) and mouse fibroblasts (3T3) were used in this study to provide two distinct mechanical properties. Cells were cultured in Ibidi microchannels (microslide VI, Ibidi) in their respective medium supplemented with $10 \%$ foetal bovine serum. 


\section{RESULTS AND DISCUSSION}

Compression testing at $2.5 \mu \mathrm{m} / \mathrm{s}$

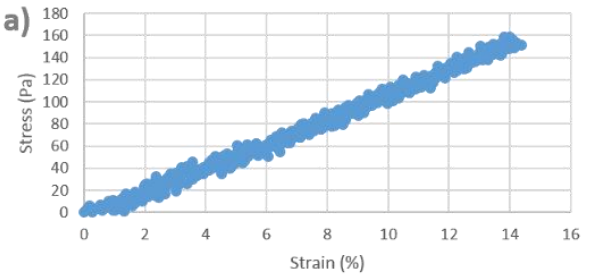

b)

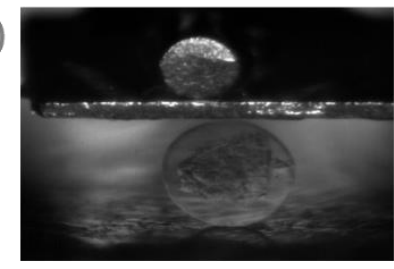

Elasticity of agarose beads

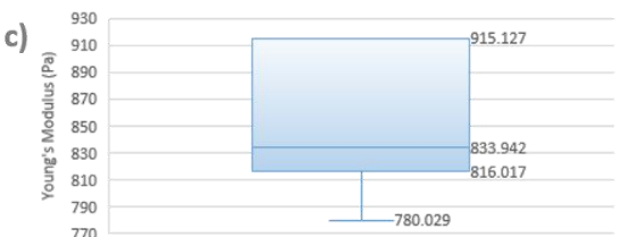

Figure 3: a) Stress-Strain curves of agarose beads, b) Compression testing of 6\% agarose bead, and c) Young's Modulus of agarose beads at $10 \%$ compression as measured in water bath at strain rate of $2.5 \mu \mathrm{m} / \mathrm{s}$.

Figure 3.a shows the stress-strain curve of 6\% agarose beads (Agarose bead technology, Madrid, Spain) acquired from compression tests using the Cell Scale Microsquisher system. Then, the mean Young's modulus, Fig 3.c, was determined to be $834 \mathrm{~Pa} \pm 45.24 \mathrm{~Pa}$ at $10 \%$ nominal compression of the bead. Single beads were tested in a water bath. This helped us to confirm that the mechanical properties of agarose beads were in the same order of magnitude as of biological cells that typically range in the $1 \mathrm{kpa}$ region. They were therefore a suitable test sample to test the new methods based on OCPM.

In Figure 4 we report the mean intensity map (Fig 4.a) and phase response of agarose beads measured using the novel OCPM system. The period of a 10kpa hydrostatic pressure was varied and we recorded a corresponding change in the time varying phase. This demonstrated that OCM could monitor nanoscale displacements induced by hydrostatic pressure in materials with mechanical properties comparable to biological cells; and therefore could be used to map the relative mechanical properties of cells in a non-invasive and real-time manner.

In the future, we will combine experimental data from fig. 3 and fig 4 . to develop a theoretical model that will convert the relative displacement measurements of this system into a quantitative measure of the cell Young's modulus.

a)

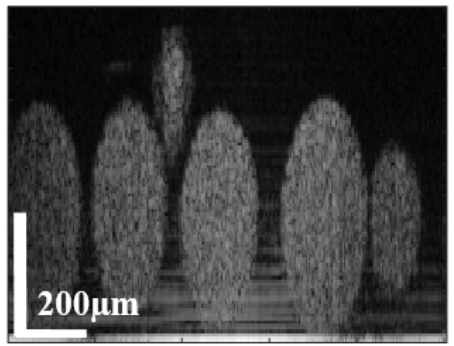

c)

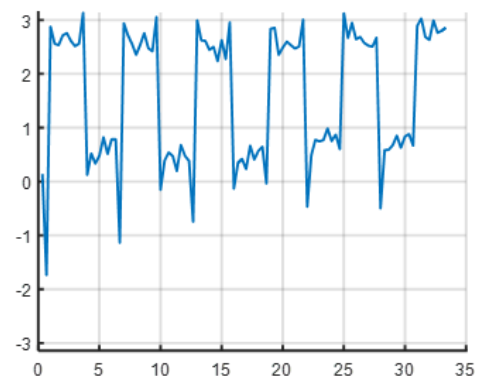

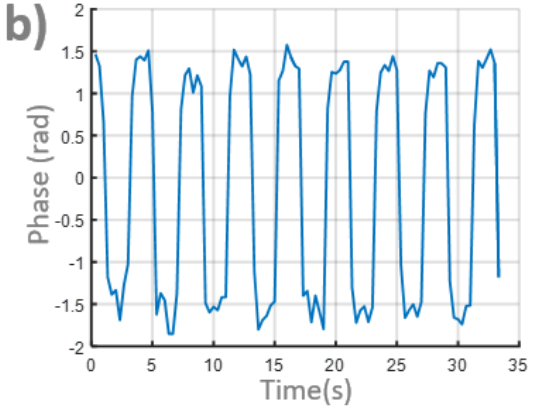

d)

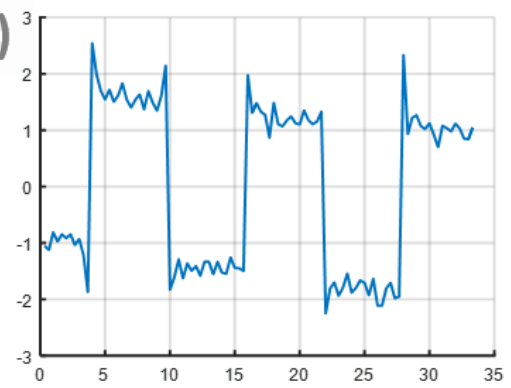

Figure 4: a) OCPM cross section of agarose beads, b-d) response to hydrostatic pressure of 4s, 6s, 12 s cycles with 100mbar amplitude. 
Cyclic stress was successfully applied directly to cells within the microfluidic chip and the corresponding displacement was recorded in real-time at the nanometre scale for each pixel of the cell (see Fig. 5). A change in amplitude and/or frequency of the stimuli was translated to a corresponding cell response. This is clearly visible comparing Fig. $5 \mathrm{~d}$, e, and $\mathrm{f}$, where we can see the change in response to varying frequency of stimuli, or when comparing Fig. $5 \mathrm{~g}$, h, and I where the amplitude of the stimuli was varied. Differences were observed between the cell lines under investigation when comparing displacement distribution. Figure 5 describes the response of MCF-7 cells. In order to further validate the ability of our platform to monitor relative biomechanical properties, MCF-7 cells were exposed to 10 $\mu \mathrm{M}$ Cytocalasin-D, an actin polymerisation inhibitor known to reduce cell stiffness (Ellridge et al., 2016). This was added 180 minutes prior to testing. Cells were imaged before and after this addition and the mean RMS of the phase signal was calculated over all cell pixels. A significant increase in cell response to hydrostatic stimulus was observed, as expected. This demonstrated the potential of OCPM combined with hydrostatic pressure to monitor non-destructively and in real-time cell mechanical behaviour.

3T3 cells have previously been described as stiffer that MCF-7 in (Rother et al., 2014). We demonstrate in Figure 6 that our system shows a marked difference in the relative displacement of 3T3 cells as compared to MCF-7 cells. Fig 6. a, e show en-face live imaging of 3T3 and MCF-7 cells respectively. Interferences between the reflections from the cell membrane and the glass surface generates "spatial" coherent interference fringes in intensity when cell thickness is below coherence gate $(<5 \mu \mathrm{m}$ in this case), hence the banding effect observed. OCT profiles of these cells are shown in fig $6 \mathrm{~b}$, g, which are then shown as the relative cell displacement in c, g. Fig $6 \mathrm{~d}$ and h show the relative change in displacement is calculated for each pixel in the cell, with 3T3 cells show a low level of displacement indicating stiffness, as compared to MCF-7 cells which show a larger mean displacement indicating a softer cell. This evidence indicates that this novel nondestructive method is capable of providing a qualitative description of cell mechanical behaviour.

a)

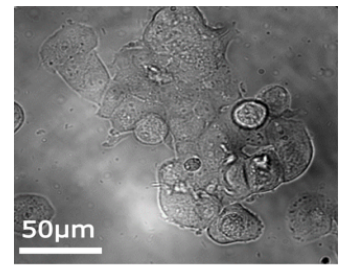

b)

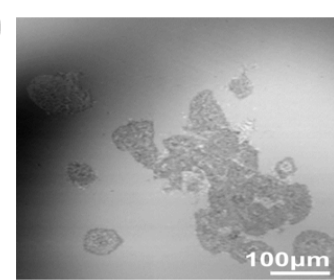

c)

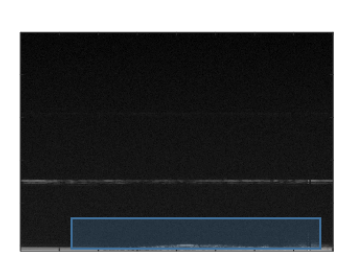

d)

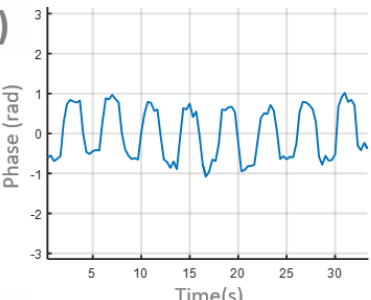

g)

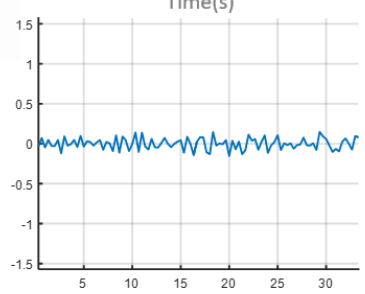

j)

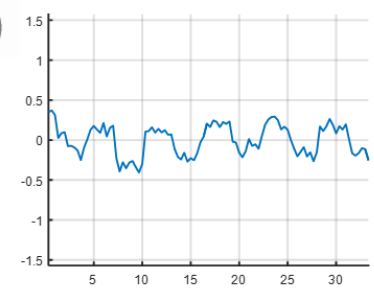

e)

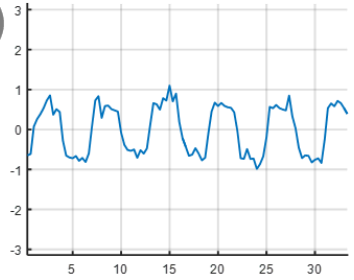

h)

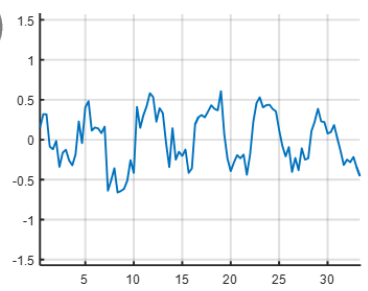

k) 1

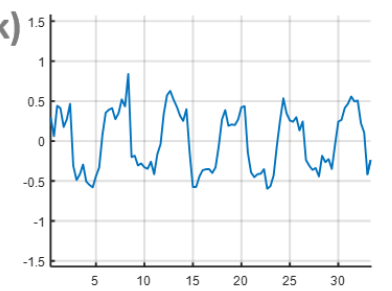

f)

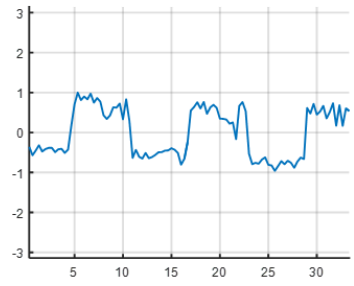

i)
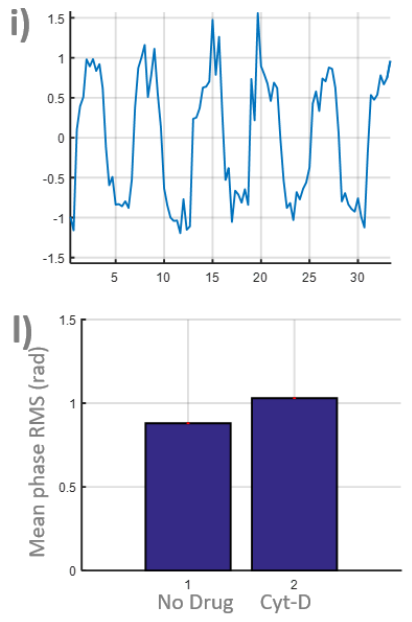

Figure 5: Bright field image (a), OCPM en-face (b), and OCPM 'cell profile' (c) of MCF-7 cells. Cell response for various hydrostatic pressure period, $4 s, 6 s, 12 s$ cycles with 200mbar amplitude $(d, e, f)$, and at 0,100, 200mbar amplitude $(g, h, i)$ with a $6 s$ cycle. (j) Typical cell response before drug addition (6s, 50mbar) and after addition of 10 $\mu M$ Cytochalasin-D ( $k$ ). (l) Mean response $(N=242$ pix) shows significant $(p<0.01)$ increase in cell response. 

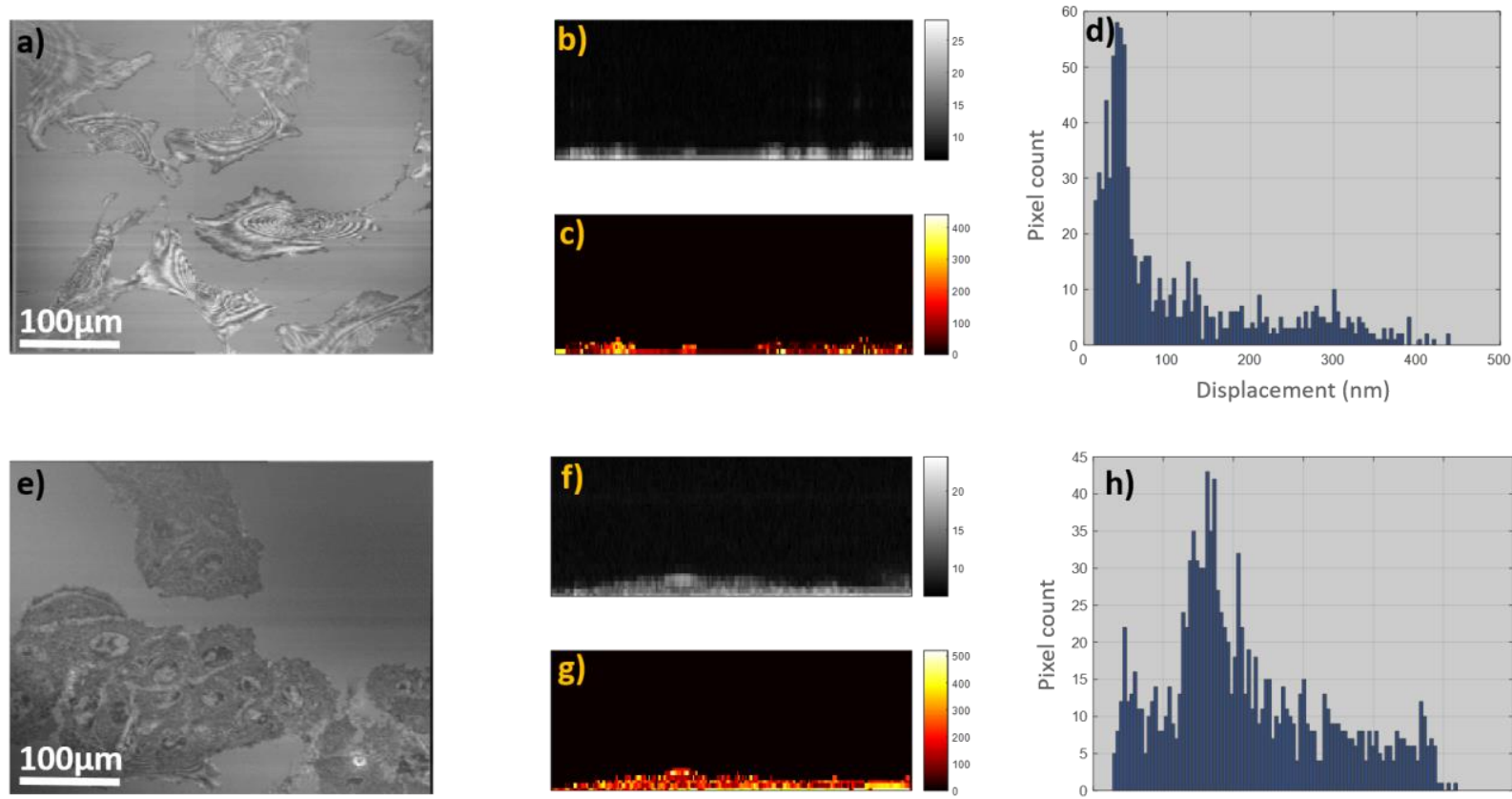
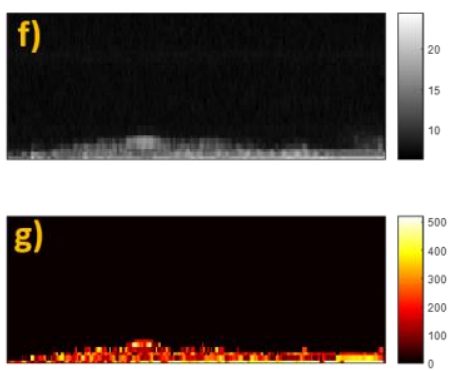

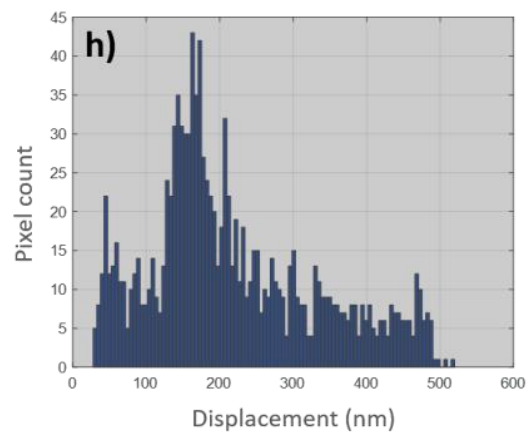

Figure 6: OCPM en-face live imaging of $3 T 3$ cells (a) and MCF-7 cells (e) with typical OCPM cross-section, 'cell profile', (b) and (f) and associated relative cell displacement induced by hydrostatic pressure (c) and $(\mathrm{g})$. Heterogeneity in intracellular displacement was found $(d, h)$ with a marked difference between $3 T 3$ and MCF-7 which suggested $3 T 3$ being stiffer.

\section{CONCLUSIONS}

We described a new method to monitor in real-time and non-destructively the mechanical properties of cells in monolayers and 3D gels that is directly translatable to the study of the mechanical behaviour of the stem cell niche.

We have monitored cell response to cyclic hydrostatic pressure. Nanoscale intracellular displacements were recorded as a function of pressure and can be directly related to the biomechanical properties of cells. Differences were observed in relative strain rates between the cell lines under investigation.

\section{FUTURE WORK}

We will use data obtained from both the Cell Scale Microsquisher ${ }^{\circledR}$ and OCPM systems in order to develop a theoretical model which will allow us to retrieve the cell Young's modulus using OCPM.

\section{ACKNOWLEDGEMENTS}

The authors are grateful to the research group of Dr Anthony Callanan in the Institute for BioEngineering, University of Edinburgh, Edinburgh, UK for facilitating use of the Cell Scale Microsquisher ${ }^{\circledR}$ system.

The authors would also like to thank the UKRMP hub for engineering and exploiting the stem cell niche, the MRC, and EPSRC for funding. 


\section{REFERENCES}

Kennedy, B. F., Kennedy, K. M. and Sampson D. D., “A Review of Optical Coherence Elastography: Fundamentals, Techniques, and Prospects”, IEEE J. Sel. Topics Quantum Electron., 20(2) 272-288(2014a)

Kim, E. K. F., Darling, E. M., Kulangara, K., Guilak, F. And Leong, K. W., "Nanophotography-induced changes in focal adhesions, cytoskeletal organisation, and mechanical properties of human mesenchymal stem cells", Biomaterials, 31(2010) 1299-1306(2009)

Kennedy, B. F., McLaughlin, R. A., Kennedy, K. M., Chin, A. C., Tien, A., Latham, B., Saunders, C. B., and Sampson, D. D., "Optical coherence micro-elastography: mechanical-contrast imaging of tissue microstructure", Biomed. Opt. Express, 5(7) 2113-2124(2014b)

Ellerbee, A. K., Creazzo, T. L. and Izatt, J. A., "Investigating nanoscale cellular dynamic with cross-sectional spectral domain phase microscopy" Opt. Express, 15(13) 8115-8124(2007)

Two-dimensional and three-dimensional viability measurements of adult stem cells with optical coherence phase microscopy.Bagnaninchi, Pierre O., Holmes Christina, Drummond Nicola, Daoud Jamal, and Tabrizian Maryam , J Biomed Opt, 2011 Aug, Volume 16, Issue 8, p.086003, (2011)

Holmes, C., Tabrizian, M. And Bagnaninchi, P. O., "Motility imaging via optical coherence phase microscopy enables label-free monitoring of tissue growth and viability in 3D tissue-engineering scaffolds", J. Tissue Eng. Regen. Med., 9(5) 641-645(2015)

Engler, A. J., Sen, S., Sweeney, H. L. and Discher, D. E., "Matrix elasticity directs stem cell lineage specification", Cell, 126(4) 677-89(2006)

Elldridge, W. J., Sheinfeld, A., Rinehart, M. T. And Wax, A., "Imaging deformation of adherent cells due to shear stress using quantitative phase imaging”, Opt. Lett., 41(2) 352-355(2016)

Rother, J., Nöding, H., Mey, I. And Janshoff, A., “Atomic force microscopy-based microrheology reveals significant differences in the viscoelastic response between malign and benign cell lines", Open Biol, 4(5) 140046(2014) 